\title{
Extracellular Vesicles and Circulating Fetuin-Mineral Complexes in Vascular Calcification; Studies by EM Techniques
}

\author{
Anjos L. Macedo ${ }^{2}$, Carla S. B. Viegas ${ }^{1}$, António P. A. Matos ${ }^{3}$ and Dina C. Simes ${ }^{1}$ \\ ${ }^{1}$ Centre of Marine Sciences (CCMAR), University of Algarve, 8005-139 Faro, Portugal. \\ 2.UCIBIO-Requimte, Departamento de Química, Faculdade de Ciências e Tecnologia, Universidade \\ Nova de Lisboa, 2829-516 Caparica, Portugal. \\ ${ }^{3 .}$ CiiEM - Centro de Investigação Interdisciplinar Egas Moniz, Campus Universitário, Quinta da Granja, \\ Monte de Caparica, 2829-511 Caparica.
}

Vascular calcification (VC) is an active and regulated multifactorial process highly relevant in the general population although currently there are no therapies available to slow or prevent the calcification progression.

Although many aspects concerning the pathogenesis of VC are still unclear, pathological features, such as chronic inflammation, increased extracellular matrix (ECM) remodeling, proliferation and differentiation of resident vascular smooth muscle cells (VSMCs), loss of anticalcific mechanisms, and release of calcifying extracellular vesicles (EVs) contribute to the development of calcific lesions [1]. It is currently accepted that the release of calcifying EVs by VSMCs, capable of efficiently nucleate hydroxyapatite in the absence of calcification inhibitors, like Fetuin-A, a potent circulating calcification inhibitor, forms the first calcification nidus in the ECM. Also, another link between VC and the presence of Fetuin-A has been suggested due to the formation of a Fetuin-mineral complex (FMC) in circulation, leading to decreased levels of free circulating Fetuin-A that otherwise would prevent calcification at the vascular wall [2].

Our purpose is to study the relationship between $\mathrm{FMC}$ formation and $\mathrm{VC}$, and to unveil the possible interplay between FMC and EV biogenesis in the vascular system.

FMC has been described to be composed of Fetuin-A containing minerals and additional calciumregulatory proteins [2]. The identification, classification and characterization of EVs requires a combination of morphological and biochemical criteria, although given their small size, some subtypes of EVs currently known as exosomes, can only be visualized by electron microscopy, allowing the determination of EVs morphology and size, and direct immunodetection of target proteins. Also, the characterization of biological isolated FMCs and synthetic protein-mineral complexes (PMCs) by EM techniques, will allow further correlations between physical mineral properties and the calcification inhibitory function of target proteins.

EVs were isolated by differential ultracentrifugation at $100.000 \mathrm{xg}$ from media of aortic segments cultured under control and calcifying conditions. Obtained EVs were resuspended in PBS, fixed in $3.7 \%$ glutaraldehyde, washed twice with PBS and dehydrated with an ascending sequence of ethanol $(40 \%$, $60 \%, 80 \%$ and 98\%). Dehydrated EVs were applied into a silica substrate and dried. Samples were examined in a scanning electron microscope (Hitachi S-2400) with elemental analysis (Bruker) after chromium sputtering. For transmission electron microscopy suspensions of the particles were adsorbed onto formvar-carbon coated grids and stained with $1 \%$ aqueous uranyl acetate. Air dried grids were observed in a JEOL 1200EX transmission electron microscope. 
We were able to characterize EVs populations produced by ex-vivo model of aortic rings [3] in calcifying conditions, using SEM. Figure 1 shows an example of the detected small vesicles with an average size of $65 \pm 12 \mathrm{~nm}$. EDS analysis of isolated EVs indicates the presence of calcium. Morphological and biochemical characterization of PMCs synthetized in the presence of different combinations of calcification inhibitory proteins is compared with serum isolated FMCs, through a combination of SEM, EDS, and TEM techniques (Figure 2). Based on morphological and size properties and differential calcium/calcium crystals detection or loading, distinct EVs populations released from aortic tissues were characterized.

Moreover, the comparison between chemical and structural composition of in vitro produced PMCs, with biological FMC entities, will bring new important information on the vascular calcification processes at molecular level.

\section{References:}

[1] R. Shroff, D.A. Long and C. Shanahan, J. Am. Soc. Nephrol. 24 (2013) p. 179.

[2] T. Hamano et al., J. Am. Soc. Nephrol. 21 (2010) p. 1998.

[3] C.S. Viegas et al., Arterioscler. Thromb. Vasc. Biol. 35 (2015) p. 399.

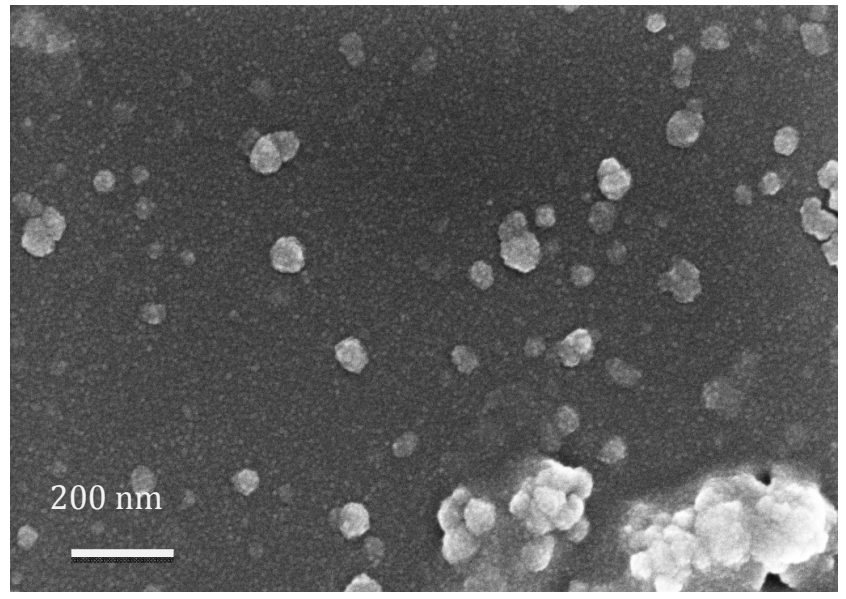

Figure 1. SEM image of isolated EVs produced by ex-vivo model of aortic rings showing a homogenous population of small vesicles $(<100$ $\mathrm{nm})$.

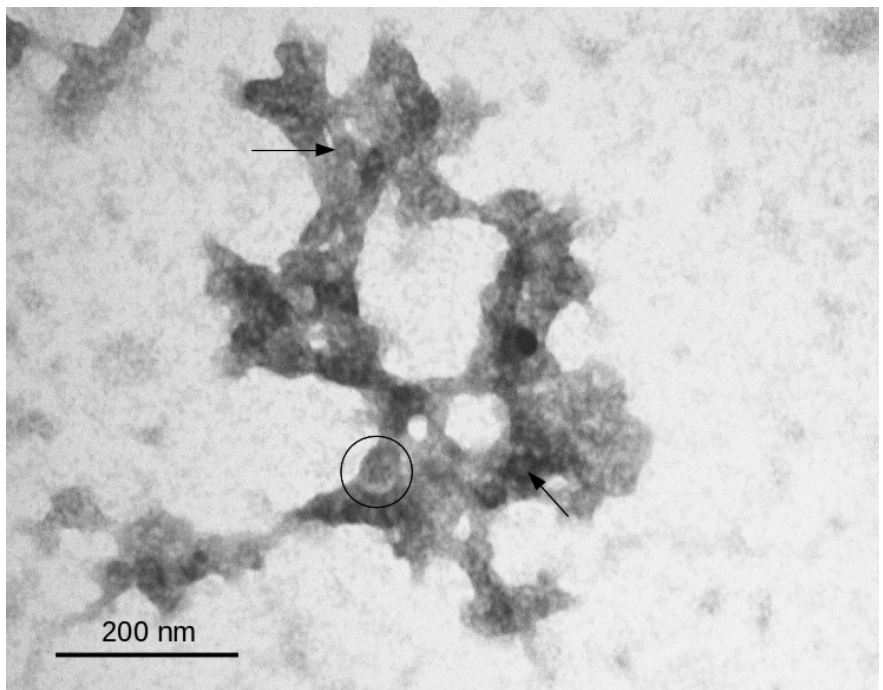

Figure 2. Negative staining of a human serum sample centrifuged at $100.000 \mathrm{xg}$ showing a suspension of particles and vesicles embedded in a putative protein containing matrix. Circle - vesicle; arrows - granules. 